\title{
Broadbent's Cognitive Approach and Its Effect on Motor Performance in Sports
}

\author{
Mehmet Koyuncu \\ Psychology Department, Faculty of Letters, Ege University, Izmir, Turkey. \\ Email: mehmet.koyuncu@ege.edu.tr \\ Received April 12 ${ }^{\text {th }}$, 2011; revised May 23 ${ }^{\text {rd }}$, 2011; accepted June 28 $8^{\text {th }}, 2011$.
}

\begin{abstract}
This study aimed to test Broadbent's attentional filtering theory in the perceptual motor task of dart throwing. Dart board size was manipulated in order to reduce the amount of information to be filtered in the participants' field of view. Sample consisted of 122 college students (63 males and 59 females) ranging in age from 17 to 36 . Participants' task was to throw 18 darts at the center of targets $45 \mathrm{~cm}, 30 \mathrm{~cm}$ and $15 \mathrm{~cm}$ in diameter. Performance was measured as radial distance from the bulls-eye of each dart. One way ANOVA, Repeated Measure of ANOVA was used in the analysis of the obtained data. The results of our study showed that dart throwing performance gave better results in cases where target's field of view was reduced, compared to the ones in which target's field of view was increased. Consistent with Broadbent's central claim, results showed that fewer stimuli in the field of view required less processing, thus, better performance. Also, those subjects that were exercising regularly did better than the ones that were not exercising. This study provides evidence that reducing target's field of view in dart throwing increases the chances to obtain better results.
\end{abstract}

Keywords: Attentional Filtering, Perception, Dart Throwing

\section{Introduction}

This study is concerned with the role attention plays in improving dart-throwing accuracy. Although dart throwing is essentially based on individual's motor skills and practice, another key factor is perception. Perception as a concept is strongly related to attention. Previous studies examining individual differences in perceptual-motor skills, such as dart throwing, have largely focused on gender, physical characteristics, colour, circadian rhythm, distance to target, metamotivational dominance and attentional focus. Results from these studies indicated that some of these factors may possibly affect dart throwing accuracy which depends on perceptual motor skills. For example, Edwards, Waterhouse, Atkinson and Reilly (2007) found that long distance dart throws improve significantly during daytime and the positively correlated intra-aural temperature. These finding indicates an association between physiological parameters and dart throwing performance.

Another factor that might be effective in dart throwing performance is colour. In a study by Eason and Smith (1980), it was found that individuals who aimed at a white-achromatic target were better able to perform dart throwing task than individuals who aimed at multi-chromatic target. However, Araki and Huddleston (2002) found no colour effect on dart throwing performance and rejected the notion which suggested that target's colour may have an effect on dart throwing accuracy. Gender differences also seem an important factor on dart throwing performance. Duffy, Ericsson, and Baluch (2007) found large sex differences in throwing accuracy even after control for physical characteristics differences.

According to Bindarwish and Tenenbaum (2006), metamotivational states can effect efficacy beliefs and performance during a motor task. They found that paratelic-dominant subjects, who are more prone to pursue goals that are perceived to be fun, were more self-efficacious and exhibited better performance in dart throwing task than telic-dominant subjects, who are more prone to pursue goals that are perceived to be important.

These individual differences cannot be conceived of as independent from attention. Dart throwing as a perceptual motor skill requires considerable amount of attention. Attention and attentional processes pervade virtually all aspects of perception, cognition, and action indeed, it is difficult to conceive of any aspect of human skill that is not, in some way, either dependent on or influenced by attention. Equally, it is difficult to conceive of any aspect of psychology that may be more central to the enhancement of skill learning and expert performance than attention (Rogers, Rousseau, \& Fisk, 1999; Abernethy, Maxwell, Masters, Van Der Kamp, \& Jackson, 2007).

In a perceptual motor skill, such as dart throwing, attention plays an important role since such goal-directed behavior requires a high degree of selectivity, concentration, and focusing at some point in the processing stream. However, our senses are affected by a variety of stimuli, either related or unrelated with the task at hand. According to D. E. Broadbent's (1958) selective filter theory, these stimuli which are not related to the task remain unattended; only basic physical properties are analyzed. Broadbent's selective filter mechanism operates in terms of stages. Initially, all stimuli are processed to extract their physical properties which are, then, stored in the immediate memory. A further processing of the stimuli as relevant or irrelevant, however, is subject to severe capacity limitations. Broadbent claims that a selective filter is needed to select certain stimuli for a further processing and to filter out other, irrelevant stimuli (Lachter, Forster, \& Ruthruff, 2004).

In their essay Forty-Five Years after Broadbent (1958), La- 
chter, Forster and Ruthruff (2004) claimed that selective filter theory of attention, championed first by Broadbent (1958), was still valid. It has been shown that more salient objects or features are more likely to trigger attentional modulation (Treisman, 1982). Attention enhances task-relevant information while inhibiting or filtering irrelevant signals (Desimone \& Duncan, 1995). Thus, an increase in the distracting stimulus might increase task interference, causing a decline in behavioral performance.

Considering Nelson's (1998) argument which claimed that large amount of information in the environment would overwhelm the limited-capacity of our system, it seems reasonable to suggest that Broadbent's (1958) "filter theory" can explain variability in dart throwing performance. Broadbent (1958) claimed that most stimuli were filtered through the attentional system before they could reach short-term memory, which was conceived as a limited capacity storage system.

Yantis and Johnston (1990) and, later, Miller (1991) also stated that people have a limited attentional capacity for processing visual events. Referring to Yantis and Johnston's (1990) and Miller's (1991) studies on perceptual load, Lachter, Forster and Ruthruff stated that:

When processing of the display is relatively simple, attention can be allocated to the entire display so that all elements will be processed. However, as processing becomes more complicated, it requires more capacity until, at some point, the capacity necessary to handle the entire display exceeds the amount available. Thus when a participant is asked to perform a simple task on a simple display, the entire display is processed, including any irrelevant items. However, when either the task or the stimuli becomes complicated, capacity is shifted away from irrelevant stimuli, resulting in reduced compatibility effects ( $p$. 889).

Selective attention allows only needed data to be processed by the nervous system's limited processing capacity while effectively eliminating potentially distracting data which can distract performance.

In sports tasks, such as dart throwing, effective and efficient operation of selective attentional processes is essential for skilled performance since critical cues are available only momentarily and sources of distraction abound information-processing resources.

Selective attention is frequently examined experimentally using tasks in which focusing of attention (“concentration”) to information from a specified modality, spatial location, or context is required in the face of competition from other items and sources of distraction. Similarly, in dart throwing, concentration and focusing are essential for a better performance. In such tasks, the selective attention of experts is frequently examined using approaches such as cue occlusion and eye movement recording (Abernethy, Wann, \& Parks, 1998) and interpretation is heavily influenced by Gibsonian notions of the education of attention and attunement (Beek, Jacobs, Daffertshofer, \& Huys, 2003; Gibson, 1991).

Another major role attention plays in human performance relates to the management and allocation of limited information-processing resources. Understanding this role involves consideration of the attentional requirements of different tasks, individual- and expertise-related differences in the capacity to divide and switch attention between concurrent tasks, and to automatize at least some task components such that they come to require little or no conscious attention to control (Rogers, Rousseau, \& Fisk, 1999; Abernethy, Maxwell, Masters, Van Der Kamp, \& Jackson, 2007).

Radlo et al. (1999) stated that external focus of attention was more facilitating than internal focus of attention in dart throwing performance. Emanuel, Jarus and Bart's (2008) results also showed that external focus of attention was related with better dart throwing performance especially in adults rather than children. They claimed that by trying to consciously control their movements (internal focus), instead of focusing on the movement effect (external focus), participants constrained their motor systems which inadvertently disrupted automatic processes. This shows that most sport skills are performed with contributions from both controlled and automatic processes, rather than one process exclusively. They depend both on skill level or stage of learning and on the nature and constraints of the task (Anson, Elliot, \& Davids, 2005; Bernstein, 1996). Assuming that controlled processing, but not automatic processing, relies heavily on the availability of a limited-capacity attentional resource (i.e., working memory), it follows that skilled performance depends on either efficient allocation of conscious attentional resources or automatization of certain subcomponents (Abernethy et al., 2007). Thus, performers will benefit from the fewer loads on the limited capacity of their attention.

Based on these arguments, we postulated that performance in dart throwing task would give better results when target's field of view was reduced (less visual stimuli to be processed), compared to an increased field of view which would contain more visual stimuli or distracting or irrelevant material to be processed. In other words, the aim of the study is to find out the effect of processing less or more visual stimuli on dart throwing performance.

\section{Method}

\section{Participants}

Participants included were 60 regular exerciser and 62 non exerciser college students with a mean age 22.16. Of all the participants 59 were female and 63 were male. Individuals representing regular exercise group were randomly selected from the School of Physical Education and Sports and individuals representing non exercise group were randomly selected from the Faculty of Letters. Participants were also asked to rate their exercise frequency in order to confirm their athletic status. They had no previous experience on dart throwing task.

\section{Procedure}

Participants were allowed five warm up dart throwing trials in order to become familiar with the task. Participants' dart throwing accuracy was evaluated under three different conditions.

There were 3 discrete blocks presented to the participants in a counterbalanced order. In the first condition (condition A), participants threw six darts to a $45 \mathrm{~cm}$ diameter dart board from $2.37 \mathrm{~m}$ distance. Dartboards were placed at $1.70 \mathrm{~m}$ height. During the first condition participants were able to see the entire $45 \mathrm{~cm}$ diameter target.

In the second condition (condition B), dartboard's size was reduced to $30 \mathrm{~cm}$ by covering its surface with a black circle 
material $15 \mathrm{~cm}$ from outside to inside (see in Figure 1). This time participants threw six darts to new $30 \mathrm{~cm}$ diameter target from the same distance.

In the third condition (condition C), participants' task was to throw six darts to $15 \mathrm{~cm}$ diameter target which was narrowed by the same method. Performance was measured as radial distance from the bulls-eye of each dart, which was considered a measure of "accuracy"-the smaller the distance, the greater the accuracy.

The posture and throwing techniques the participants adopted were of their on choosing, but they were required to maintain these in all of three conditions. Participants were instructed always to aim for the bull eye.

\section{Design}

Three independent variables were tested in the experiment by a between subject design. The efficacy of the conditions (Condition A, Condition B, and Condition C) and groups (regular exerciser and non exerciser) was tested by a $3 \times 2$ between subject design.

\section{Data Analysis}

In the analysis of obtained data set descriptive statistics, such as Independent Samples $\boldsymbol{t}$-test, One Way ANOVA, Repeated Measure ANOVA, were used. Data was analyzed with SPSS 11.0 (SPSS, Inc., Chicago ILL, USA).

\section{Results}

The experiment included 122 participants and the descriptive statistics results of regular and non-exercisers were shown in Table 1.

In order to examine whether there is a difference in dart throwing accuracy based on target size (3) and regular exercise participation (2) Repeated Measure of ANOVA was conducted. Results indicated that there were significant differences within

Table 1.

Descriptive statistics results of regular and non exercisers.

\begin{tabular}{lcccc}
\hline & $\begin{array}{c}\text { Regular } \\
\text { Exercisers }\end{array}$ & \multicolumn{3}{c}{$\begin{array}{c}\text { Non } \\
\text { Exercisers }\end{array}$} \\
\hline Condition A & Mean & S.d. & Mean & S.d. \\
Condition B & 9.16 & 3.81 & 9.87 & 3.01 \\
Condition C & 8.47 & 2.74 & 9.75 & 3.61 \\
\hline
\end{tabular}

subject effect of targets size $\left(\mathrm{F}_{2,117}=3.656 ; p<.05, n^{2}=.059\right)$. Thus, there were significant accuracy differences between condition $\mathrm{A}$ (mean $=9.55$, sd $=3.44)$, and condition $\mathrm{C}($ mean $=$ 8.57, sd = 3.83) indicating that $15 \mathrm{~cm}$ diameter target dart throwings (condition $\mathrm{C}$ ) revealed significantly better performances than $45 \mathrm{~cm}$ diameter target dart throwings (condition A). Repeated measures of ANOVA showed that there were not any significant differences between subject effect in dart throwing accuracy $\left(\mathrm{F}_{2,117}=.348 ; p>.05, n^{2}=.006\right)$.

To validate repeated measure's result, which indicated that exercise participation had no effect on dart throwing accuracy, Independent Sample $\boldsymbol{t}$-test was carried out. Results showed that regular exercisers (mean $=8.47$, sd $=2.74$ ) had significantly better dart throwing accuracy than non-exercisers (mean $=9.75$, $\mathrm{sd}=3.61)$ only in condition B (t $(120)=2.16, p<.05)$ while no accuracy differences were noticed between regular exercisers and non-exercisers in Condition A and C.

Independent Sample $\boldsymbol{t}$-test was conducted to see the gender effect on dart throwing accuracy. Results revealed that males had greater dart throwing accuracy than females in all of the three conditions (Table 2).

To examine the effect of height as a physical trait on dart throwing accuracy, participants were divided into 3 groups. Individuals measuring $169 \mathrm{~cm}$ or lower were included in short height group; medium height group consisted of individuals measuring between 170 - $177 \mathrm{~cm}$ in height; $178 \mathrm{~cm}$ and upper individuals were included in the tall group.

One way ANOVA was conducted in order to see if any significant accuracy differences existed among height groups. One way ANOVA with Post Hoc Scheffe indicated that taller participants' dart throwing performance was better than shorter participants' performance in Condition A, B, and C. In addition, medium height participants had greater dart throwing accuracy than shorter participants in condition C (Table 3).

Table 2.

Independent sample t-test on gender effect for dart throwing accuracy.

\begin{tabular}{lcccccc}
\hline & Groups & $n$ & Mean & Std. Deviation & $t$ & $p$ \\
\hline \multirow{2}{*}{ Condition A } & Female & 59 & 10.57 & 3.74 & \multirow{2}{*}{3.41} & .001 \\
& Male & 63 & 8.54 & 2.79 & & \\
Condition B & Female & 57 & 10.38 & 3.64 & & \\
& Male & 63 & 8.02 & 2.45 & & .000 \\
Condition C & Female & 59 & 10.21 & 4.44 & & \\
& Male & 63 & 7.02 & 2.40 & 4,99 & .000
\end{tabular}

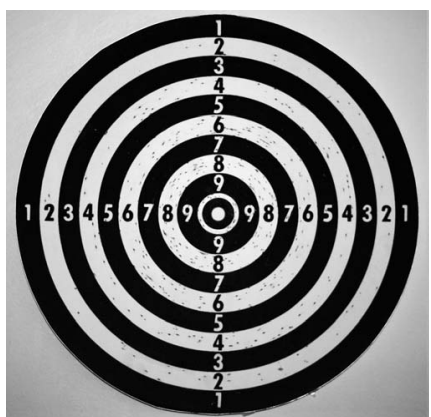

(a)

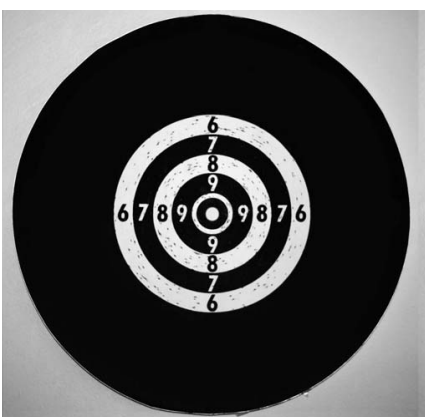

(b)

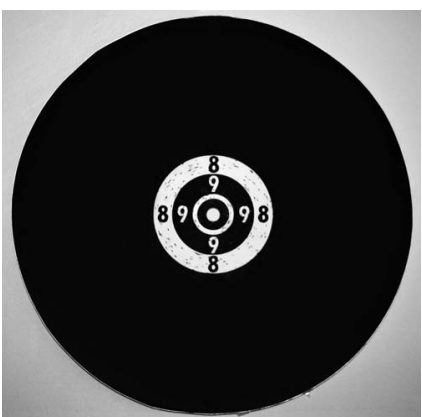

(c)

Figure 1.

Restructured for three different experimental conditions ( $a, b$, and c respectively). 
Table 3.

One Way ANOVA results on height groups.

\begin{tabular}{lccccccc}
\hline & & $N$ & Mean & St.D. & $F$ & Sig. & $n$ \\
\hline \multirow{4}{*}{ Condition A } & Short & 44 & 10.65 & 3.50 & & & \\
& Normal & 41 & 9.55 & 3.53 & 5.76 & .004 & .10 \\
& Tall & 37 & 8.16 & 2.73 & & & \\
& Short & 43 & 10.32 & 3.71 & & & \\
Condition B & Normal & 40 & 8.63 & 3.05 & 4.62 & .012 & .10 \\
& Tall & 37 & 8.34 & 2.60 & & & \\
& & & & & & & \\
Condition C & Short & 44 & 10.77 & 4.39 & & & \\
& Normal & 41 & 7.81 & 3.35 & 14.54 & .000 & .20 \\
& Tall & 37 & 6.78 & 2.18 & & & \\
\hline
\end{tabular}

\section{Discussion}

Results of the study confirmed our initial hypothesis suggesting that dart throwing accuracy would improve when the target's size was reduced. This result is consistent with selective filter theory, originally suggested by Broadbent (1958). Decreasing the unnecessary stimuli in the visual field of the participants by reducing the size of the dartboard resulted in increased quality of response and accuracy due to less information directed to the nervous system to be processed. Luck and Hillyard's (1994) argument suggesting that the human visual system is frequently confronted with complex visual scenes containing multiple objects, and accurate perception under these conditions poses significant computational problems also confirms our results.

Also, it was found that male participants had greater dart throwing accuracy than females in all of the three conditions. This result is compatible with Duffy, Balunch, and Ericsson (2004). In their study Duffy, Balunch, and Ericsson (2004) observed a gender effect on dart throwing performance even after controlling for physical attributes such as arm length.

In another study conducted again by Duffy, Ericsson, and Baluch (2007), they showed a gender difference on dart throwing performance in favor of males. As they stated differential engagement in associated motor activities may explain gender differences in dart throwing accuracy. Results about gender difference in our study, may also be an evidence verifying Hodges, Huys, and Starkes' (2007) findings. They argued that "practice experiences as a function of gender are both quantitatively and qualitatively different, with the demands and amount of practice for women athletes being more stringent than those for men to attain a similar level of performance.” Another possible explanation for gender differences in dart throwing may be endocrine system activities. As Gouchie and Kimura (1991) demonstrated, higher level of salivary testosterone was associated with better performance on spatial ability task in both males and females.

The results of the study showed that there were performance differences in dart throwing in terms of height. Thus, taller participants were better able to perform on dart throwing task than shorter participants. These results were similar to Duffy, Ericsson, and Baluch's (2007) results. In their regression model, height could significantly predict dart throwing performance in expert dart players.

No differences occurred between the dart throwing perform- ances of regular exercisers and non-exercisers. This is important in demonstrating that regular exercising did not have any significant effect on dart throwing accuracy. However, manipulation of the dart board's size (that is, reducing the visual field and, consequently, inhibiting distracting stimuli) did produce significant differences.

Further investigation could be conducted in sports using different throwing styles and throwing material, such as archery, to test the effect of stimulus type (less or more stimuli) on the performance of the participants. Also, choosing a colored target material instead of a black and white dart board might prove interesting results.

\section{References}

Abernethy, B., Wann, J., \& Parks, S. (1998). Training perceptual motor skills for sport. In B. Elliott (Ed.), Training in sport: Applying sport science (pp. 1-55). London: Wiley Publications.

Abernethy, B., Maxwell, J. P., Masters, R. S. W., Van Der Kamp, J., \& Jackson, R. C. (2007). Methodological review and evaluation of research in expert performance in sport. In G. Tenenbaum and R. C. Eklund (Eds.), Handbook of sport psychology (pp. 161-184). Hoboken, NJ: John Wiley \& Sons, Inc.

Anson, G., Elliott, D., \& Davids, K. (2005). Information processing and constraints-based views of skill acquisition: Divergent or complementary? Motor Control, 9, 217-241.

Araki, K., \& Huddleston, S. (2002). The effect of color on a target accuracy task. International Sports Journal, 6, 86-92.

Beek, P. J., Jacobs, D. M., Daffertshofer, A., \& Huys, R. (2003). Expert performance in sport: Views from the joint perspectives of ecological psychology and dynamical systems theory. In J. L. Starkes and K. A. Ericsson (Eds.), Expert performance in sports: Advances in research on sport expertise (pp. 321-344). Champaign, IL: Human Kinetics.

Bernstein N. A. (1996). On dexterity and its development. In: M. L. Latash and M. T. Turvey (Eds.), Dexterity and its development (pp. 3-224). Hillsdale, NJ: Lawrence Erlbaum Associates.

Bindarwish, J., \& Tenenbaum, G. (2006). Metamotivational and contextual effects on performance, self-efficacy, and shifts in affective states. Psychology of Sport and Exercise, 7, 41-56. doi:10.1016/j.psychsport.2005.04.001

Broadbent, D. E. (1958, 1998). Perception and communication. London: Pergamon Press.

Cowan, N. (1998). Attention and memory: An integrated framework. Cary, NC: Oxford University Press.

Desimone, R., Duncan, J. (1995). Neural mechanisms of selective visual attention. Annual Review of Neuroscience, 18, 193-222. doi:10.1146/annurev.ne.18.030195.001205

Duffy, L. J., Baluch, B., \& Ericsson, K. A. (2004). Dart performance as a function of facets of practice amongst professional and amateur men and women players. International Journal of Sport Psychology, 35, 232-245.

Duffy, L. J., Ericsson, K. A., \& Baluch, B. (2007). In search of the loci for sex differences in throwing: The effects of physical size and differential recruitment rates on high levels of dart performance. $R e$ search Quarterly for Exercise and Sport, 78, 71-78.

Eason, B. L., \& Smith, T. L. (1980). Effects of multi-chromatic and achromatic targets and darts on throwing. Perceptual and Motor Skills, 51, 519-522.

Edwards, B., Waterhouse, J., Atkinson, G., \& Reilly, T. (2007). Effects of time of day and distance upon accuracy and consistency of throwing darts. Journal of Sports Sciences, 25, 1531-1538. doi: $10.1080 / 02640410701244975$

Emanuel, M., Jarus, T., \& Bart, O. (2008). Effect of focus of attention and age on motor acquisition, retention and transfer: A randomized trial. American Physical Therapy Association, 88, 251-260.

Gouchie, C., \& Kimura, D. (1991). The relationship between testoster- 
one levels and cognitive ability patterns. Psychoneuroendocrinology, 16, 323-334. doi:10.1016/0306-4530(91)90018-O

Hodges, N. J., Huys, R., \& Starkes, J. L. (2007). Methodological review and evaluation of research in expert performance in sport. In G. Tenenbaum and R. C. Eklund (Eds.), Handbook of sport psychology (pp. 161-184). Hoboken, NJ: John Wiley \& Sons, Inc.

Lachter, J., Forster, K. I., \& Ruthruff, E. (2004). Forty-five years after Broadbent (1958): Still no identification without attention. Psychological Review, 111, 880-913. doi:10.1037/0033-295X.111.4.880

Luck, S. J., \& Hillyard, S. A. (1994). Spatial filtering during visual search: evidence from human electrophysiology. Journal of Experimental Psychology: Human Perception and Performance, 20, 10001014. doi:10.1037/0096-1523.20.5.1000

Miller, J. (1991). The flanker compatibility effect as a function of visual angle, attentional focus, visual transients, and perceptual load: A search for boundary conditions. Perception \& Psychophysics, 49, 270-288. doi:10.3758/BF03214311

Radlo, S. J. (1999). Effectiveness of singer's five-step strategy during competition: A psychophysiological investigation. Journal of Sport \& Exercise Psychology, 21, S88.

Rogers, W. A., Rousseau, G. K., \& Fisk, A. D. (1999). Applications of attention research. In F. T. Durso (Ed.), Handbook of applied cognition. Chichester: Wiley.

Treisman, A. (1982). Perceptual grouping and attention in visual search for features and for objects. Journal of Experimental Psychology: Human Perception and Performance, 8, 194-214.

Yantis, S., Johnston, J. C. (1990). On the locus of visual selection: Evidence from focused attention tasks. Journal of Experimental Psychology: Human Perception and Performance, 16, 135-149. doi:10.1037/0096-1523.16.1.135 